\title{
MÜNZEN AUS DER ÄLTEREN RÖMERZEIT IM MILIEU DER NORDKARPATHISCHEN GRUPPE IN DER ZIPS
}

\author{
M A R I ÁN SOJÁK ${ }^{\mathbb{D}}$ - A N DRE J N O ÁK ${ }^{1}$
}

\begin{abstract}
Coins from the Early Roman Period in the North Carpathian Group in the Spiš. The paper discusses Roman coins found separately in the outskirts of the town of Spišská Belá (Kežmarok distr.). The analysis showed that the coins are Roman denarii from the $1^{\text {st }}-2^{\text {nd }}$ C., between years 73 and 180 CE. All the coins were found at registered archaeological sites attributed to the northern Carpathian group dated back to the turn of the $4^{\text {th }}$ and $5^{\text {th }}$ c. Most likely, the coins were not in circulation so deep in Barbaricum. Instead, they had been gathered and transferred through generations, and the precious metal they were made of - silver - was later secondarily used. This hypothesis is corroborated by finds of numerous coins with broken edges including seven denarii from Spišská Belá. The secondary use of older Roman denarii by people belonging to the northern Carpathian group was confirmed at numerous sites in Spiš (e.g. the hoard from Žehra, Temná Cave) or Liptov but also in Czechia, Lesser Poland, Ukraine and Upper Silesia.
\end{abstract}

Keywords: Slovakia, North Carpathian group, Roman coins, communication.

\section{EINLEITUNG}

In den Jahren 2018 bis 2020 ist es dank einer systematischen Rekognoszierung im Kataster der Gemeinde Spišská Belá, Bez. Kežmarok (Abb. 1) gelungen, eine interessante Kollektion numismatischen Charakters zu entdecken. Hervorzuheben sind Exemplare römischer Münzen aus dem 1.-2. Jh. n. Chr., die aus folgenden archäologischen Lokalitäten stammen (Abb. 2).

1. Krivá medza - bei der Eisenbahnstrecke

Rom, Domitianus (81-96), Rom, Denar 92-93, Ag, 19 mm, 2,66 g. RIC II, 790 (Abb. 3: 1).

A: Kopf nach rechts, Lorbeerkranz, IMP CAES DOMIT AVG GERM P M TR P (XV).

R: Minerva steht links, linke Hand an der Hüfte, Lanze in der rechten, IMP XXII COS XVII CENS P P P.

2. Kahlenberg - am Fuß von Stirn

a) Rom, Traianus (98-117), Rom, Denar 103-111, Ag, 18,5 mm, 1,86 g (abgebrochener Rand). RIC II, 118 (Abb. 3: 2).

A: Kopf nach rechts, Lorbeerkranz, (IMP) TRAIANO AVG (GER DAC PM TRP).

R: Aequitas steht links, mit Füllhorn in linker Hand, Waage in der rechten, COS V PP SPQR OPTIM (O PRINC).

b) Rom, Antoninus Pius (138-161), Rom, Denar 140-143, Ag, 17,5 mm, 2,55 g, abgewetzte Oberfläche. RIC III, 64 (Abb. 3:3).

A: Kopf nach rechts, Lorbeerkranz, (ANTONINVS AVG PIVS P P TR P COS III).

R: Clementia steht links, mit Zepter in linker Hand, Opferschale in der rechten, (CLEMENTIA AVG).

c) Rom, Commodus (177-192), Rom, Denar 180, Ag, 16-18,5 mm, 1,98 g. RIC III, 264 (Abb. 3: 4).

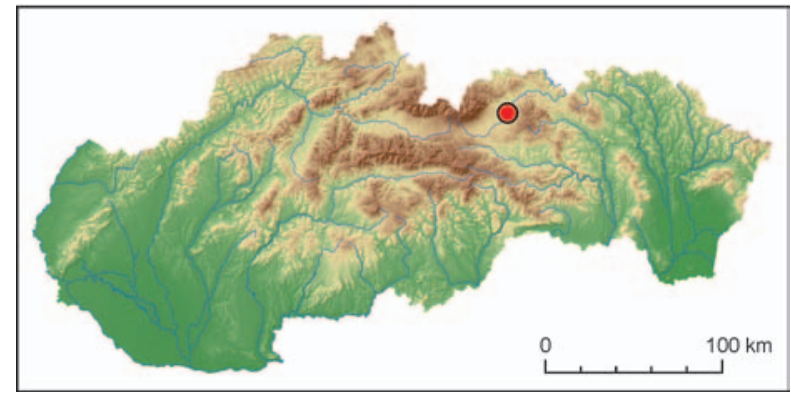

Abb. 1. Karte der Slowakei mit der Lokalisierung von Spišská Belá.

A: Lockenkopf von Marcus Aurelius nach rechts, (DI) VVS M ANTO (NINVS PIVS).

R: rechts Adler auf einer Stange stehend, schaut nach links, CONSECRATIO.

d) Rom, Faustina II. (145-180), Rom, Denar 156-161, Ag, 18-19 mm, 2,57 g. RIC III, 496 (Abb. 3: 5).

A: Kopf rechts, FAVSTINA AVGVSTA.

R: Concordia steht links, in der linken Hand ein Füllhorn, in der rechten Opferschale, (AVGVSTI PII FIL).

3. Hoher Rand

a) Rom, Vespasianus (69-79), Rom, Denar 73, Ag, $17 \mathrm{~mm}$, 1,58 g (zweimal abgebrochene Ränder, abgewetzte Oberfläche). RIC II, 546 (Abb. 3: 6).

A: umkränzter Kopf nach rechts, IMP C (AES VESP AVG CENS).

R: Kaiser sitzt auf dem Stuhl rechts, mit Zepter in linker Hand, Olivenzweig in der rechten, (PONTIF MAXIM).

b) Rom, Traianus (98-117), Rom, Denar 107-108, Ag, 19 mm, 1,49 g (zweimal abgebrochene Ränder). RIC II, 96 (Abb. 3:7).

1 Der Beitrag entstand im Rahmen des EG-Mittelprojektes Nr. 02/0101/19 und 02/0124/20 der Agentur VEGA. 


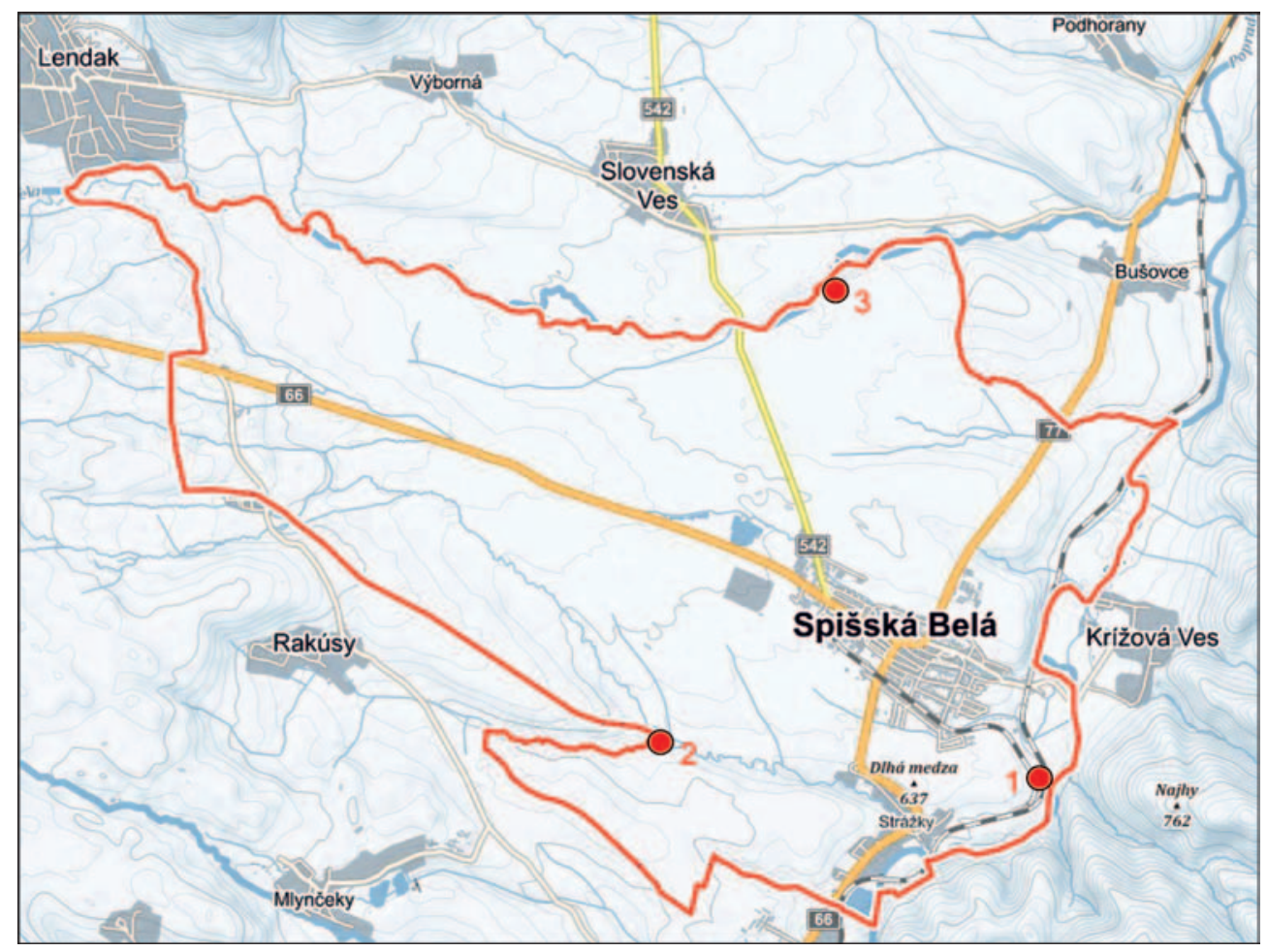

Abb. 2. Spišská Belá. Fundstätte römischer Münzen im Katastergebiet der Stadt. 1 - Krivá medza, bei der Eisenbahnstrecke; 2 - Kahlenberg, am Fuß von Stirn; 3 - Hoher Rand (nach www.mapy.cz, bearbeitet).

A: umkränzter Kopf nach rechts, (IMP T)RAIANO AVG GE(R DAC P M TR P);

$R$ : dakischer Gefangene sitzt rechts auf einem Haufen dreier Schutzschilder, auf den Seiten zwei Lanzen und zwei Schwerte, mit verbundenen Händen hinter dem Rücken, COS V P P (S P Q R OPTIMO PRINC), im Abschnitt DAC CAP.

\section{AUSWERTUNG \\ DER NUMISMATISCHEN FUNDE}

Alle präsentierten Funde der einzeln entdeckten römischen Münzen stammen aus registrierten archäologischen Fundstellen, auf welchen außer urzeitlichen Besiedlungen (vorwiegend aus dem Epipaläolithikum, Mesolithikum, Neolithikum und der Bronzezeit) auch markante Siedlungen der Nordkarpathischen Gruppe aus dem Ende der Römerzeit und Anfang der Völkerwanderung belegt sind (Pieta 1991). Aus keiner Lage sind bis jetzt bekannte Denkmäler, die genauso alt wie die datierten Münzen wären, d. h. eingegliedert in die Púchov-Kultur der älteren Römerzeit. Allgemein gibt es im derzeitigen Gemeindegebiet, bis auf bescheidene und problematisch datierte Funde aus älteren Rekognoszierungen (Kollárová/Novák/Soják 2015, 87 ff.), keine Spuren nach Besiedlung aus der ganzen Latènezeit und der älteren Römerzeit, $d$. $h$. intensive Belege der Träger der Púchov-Kultur in der Zips (Soják 2006a, 40 ff.). Die gleiche Situation wiederholt sich auch auf vielen anderen Fundstellen in der Zips, auf welchen nach dem Untergang der Púchov-Kultur erst die Besiedlung des Volkes der Nordkarpathischen Gruppe dominiert. Im Gegensatz dazu ist für jetzt die Besiedlung der PrzeworskKultur aus dem Ende des 2. bis 3. Jh. n. Chr. auf dem behandelten Gebiet für diesmal weniger markant und bindet sich bisher vorbehaltslos auf die Unterzips im Flussgebiet von Hornád (Dolany, Klčov, Levoča, Nemešany, Smižany/Spišské Tomášovce, Spišský Hrhov). Eine andere kulturelle Situation ist im Flussgebiet von Poprad in der Oberzips, wo nach der Púchov-Kultur vorläufig ein Siedlungshiatus zu verfolgen ist und die darauffolgende Besiedlung schließt an die Träger der Nordkarpathischen Gruppe an. In einen Zusammenhang mit dieser Gruppe sind die Traianus-Denare aus den Lokalitäten Vlková-Levkovce (Soják 1998), Stráne pod Tatrami (Soják 2002), wie auch Hôrka-Primovce (Soják 2004), aber vor allem der Satz der Denkmäler aus ŽehraTemná jaskyňa (Dunkle Höhle), die sich in der Travertin-Felswand von Spišský hrad (Zipser Burg) befindet, zu setzen (Soják 2005a; 2005b; 2006b). Die zuletzt erwähnte Lokalität mit Funden römischer Münzen, zwei Lederbeuteln und anthropologischen Überresten des Inhabers des Geldbetrags (Jakab 


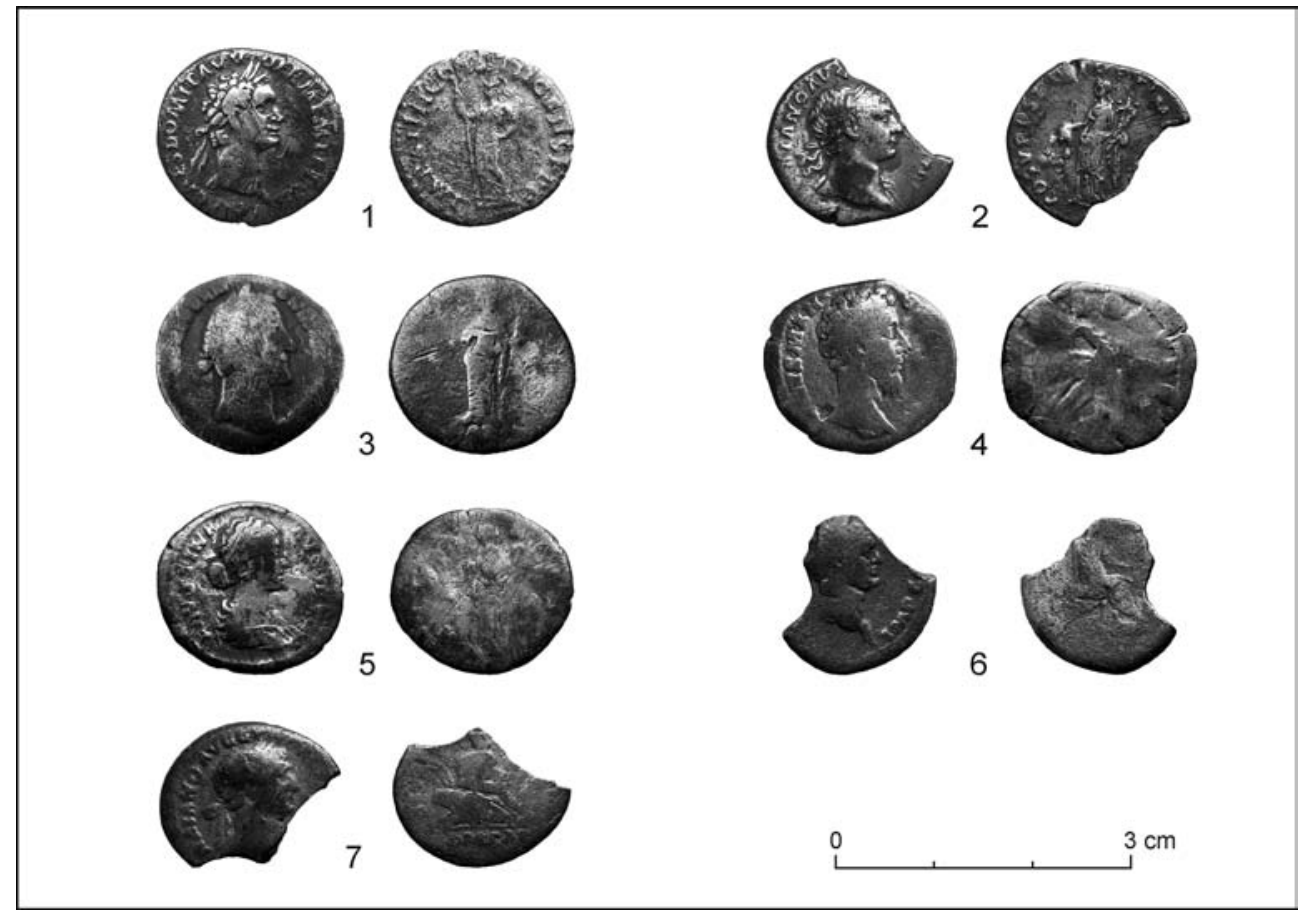

Abb. 3. Spišská Belá. Römische Denare aus Begehungen der Lagen Krivá medza, bei der Eisenbahnstrecke (1), Kahlenberg, am Fuß von Stirn (2-5) und Hoher Rand (6, 7). 1 - Domitianus; 2, 7 - Traianus; 3 - Antoninus Pius; 4 - Commodus; 5 Faustina II.; 6 - Vespasianus (Foto M. Soják).

2005) wurde früher, auch wenn mit angedeuteten Vorbehalten, mit dem Untergangshorizont der dortigen Púchov-Besiedlung aus dem Abschluss des 2. Jh. n. Chr. verbunden. Darüber zeugt der Burgwall der Púchov-Kultur, situiert auf der Anhöhe der Burgberges und mit reichen Denkmälern aus seiner ganzen Entwicklungszeit. Die neuere Radiokarbondatierung der Knochenüberreste aus Temná jaskyňa zeigte aber eindeutig die Bindung dieser Fundeinheit mit einem „spätrömischen Barbaren“.'2 Wahrscheinlich hängt mit derselben Besiedlung auch der Zufallsfund eines TraianusDenars aus Poprad (ein nicht publizierter Fund von M. Kiska in den 80-er Jahren des 20. Jh.; RIC II 1968, 169) zusammen, entdeckt im Hinterland einer naheliegenden Siedlung der Nordkarpathischen Gruppe, die auf der Flur Za Popradom lokalisiert wurde (Soják 2003a, 133). Vielleicht kann man mit dem gleichen kulturellen Milieu den Zufallsfund eines Hadrianus-Denars in Verbindung setzen, den man im Jahr 2018 hinter dem westlichen Rand der Firma Slovdekra entdeckte (ein nicht publizierter Fund; RIC II 1968, 81), hinter dem östlichen Rand der erfassten nordkarpathischen Besiedlung auf der Flur Smižianska roveň. Auf der letztmalig erwähnten Flur vermerken wir jedoch auch eine Besiedlung aus dem Ende der älteren (Ende der Púchov-Kultur) und Anfang der jüngeren Römerzeit, worüber Funde römischprovinzialen Keramik Terra Sigillata aus der 2. Hälfte des 2. Jh. bis erste Hälfte des 3. Jh. n. Chr. und in der Zips einzigartige antike Gemme aus dem vorausgesetzten 3. Jh. n. Chr. (Daňová/Soják 2013; Soják 2014, 45), zeugen. Der gemeinsame Nenner aller höher genannten und im präsentierten Beitrag analysierten Funde aus Spišská Belá, ist eine mehr oder weniger abgewetzte Oberfläche der einzelnen Exemplare, was über einen langfristigen Umlauf zeugt, bzw. über Anwendung während langer 200 bis 300 Jahre, zeugt. Die Prägungen aus Spišská Belá sind sogar absichtlich auf den Randen einmal, bzw. zweimal ausgebrochen, was über einer sekundären Gewinnung und Nutzung des kostbaren Metalls (in diesem Fall des Silbers) zur Wiederverwendung zeugt. Ein ähnlicher Fall ist die Kollektion von Denaren aus Temná jaskyňa, wo sich unter markant abgenutzten Münzen eine mit mehrfach abgebrochenem Rand befand. Gleich ist auch der Fall des fragmentarisch erhaltenen Hadrianus-Denars aus Janovce bei Bardejov, der als Zufallsfund auf dem Weg näher zur

\footnotetext{
2 Die Radiokarbonanalyse der menschlichen Überreste in Poznań wies auf ein Alter zwischen 234-381 AD hin. Die Anfänge der Nordkarpathischen Gruppe sind bisher nicht zuverlässig datiert, obwohl das Ende dieser Daten aus Temná jaskyňa hervorragend auf ihre Entwicklung passt.
} 
entgegengesetzten/nördlichen Seite der Karpaten entdeckt wurde (Soják/Fecko 2015).

Es ist wichtig zu erwähnen, dass die analysierten Münzen aus dem Kataster von Spišská Belá nicht die einzigen sind, die wir auf dem Gebiet der jetzigen Gemeinde registrieren. Dies belegen Lesefunde von Michael Greisiger, einem Zipser-Polyhistoren, aus der Wende des 19. und 20. Jh. (Minarovičová 1996; Soják 2006a, 43 mit Abb.) und auch weitere Funde größerer Zahl von Prägungen ohne näher bekannten Fundumständen (Soják 2008, 36, hier weitere Lit.). Bei den meisten Funden handelt es sich um Prägungen aus der älteren Römerzeit, die eindeutig mit der erörteten kulturellen Gruppe zusammenhängen. Das belegt vorwiegend die zahlreiche Begleitkeramik aus dominanten verzierten Vorratsgefäßen mit Krause, vereinzelt auch mit anderen zeitgenössischen Begleitartefakten einschließlich solcher, die aus Metall hergestellt wurden. Zu erwähnen sind vor allem die Gewandschließen - Fibeln aus Bronze (mit umgeschlagenem Fuß aus Vel'ká Lomnica und aus Stráne pod Tatrami, Typ Wiesbaden aus Kahlenberg/Stirn: Lipták 1935, 63, Taf. XIV unten; Novotná/Soják 2013, 30 f., Abb. 20: 1; Soják 2003, 171 ff., Abb. 5 und nicht publiziert), oder Silber (Spišská Belá, bei Mlynský ker; nicht publiziertes Fragment einer Blech-Bogenfibel, der jüngsten im Rahmen der Nordkarpathischen Gruppe). ${ }^{3}$ Viele Münzen aus den ersten zwei Jahrhunderten n. Chr. aus der Zips können genauso mit einer spätrömischen Besiedlung zusammenhängen und müssen daher nicht eine gleichzeitige Besiedlung mit deren Datierung/Umlauf indizieren. Als Beispiel sind Funde aus dem Travertin-Komplex Dreveník über Spišské Podhradie zu erwähnen, wo einzelne Exemplare römischer Münzen gefunden wurden, zusammen mit einem Depot von 60 Stücken von Münzen mit dem Bildnis des Kaisers Hadrianus aus der älteren Römerzeit (Kolníková 1972, 104; 1980, 72 - hier unter Spišské Vlachy; Šmálik 1967), sowie auch aus weiteren Zipser Fundstellen (Soják 2000; 2008, 34 ff.).

\section{ZUSAMMENFASSUNG}

Die Interpretation der Funde römischer Münzen ist kompliziert und hängt von vielen Faktoren ab, in Abhängigkeit von Zufallsfunden oder Depots, Fundumständen, der Zusammensetzung der geretteten Kollektionen, die oft aus Depots mit einer großen Zeitspanne bestehen (Kolníková 1972; 1973). Deshalb überrascht nicht im Barbaricum, so weit von Limes Romanus entfernt, schon seit längerer Zeit angesammeltes Vermögen in Form von Münzen aus relativ feinem Silber. Diese wurden von Generation zu Generation übergeben und bereitgestellt zur sekundären Nutzung jahrzehntelang nach der Ausscheidung der gültigen Umlaufswährung. Die Funktion der römischen Münzen als gesetzliches Umlaufsgeld ist auf dem Gebiet des Römischen Reichs wie in der Nähe des römischen Limes gültig. Im Gegenteil, weit in Barbaricum, hatten sie ohne Zweifel eine andere Bedeutung, ob als Erinnerungsgegenstände, die aus Kriegszügen von Veteranen gebracht wurden, oder durch einen breit verzweigten Handel, der durch die Zips bis auf das Territorium des heutigen Polens verlief, der sich vielleicht dank prosperierendem Handel mit Eisenrohstoff entwickelte. In den Funden überwiegen Denare aus dem 2. Jh. n. Chr., die die markanteste Gruppe der römischen Münzen in dem ganzen barbarischen Milieu (Bursche 1994), einschließlich des slowakischen Gebiets (Kolníková 1994), bilden. Den bisherigen Funden nach, war ihr Hauptzufluss im Verlauf der turbulenten Ereignisse der Markomannenkriege, was durch vorherrschende Prägungen mit dem Abbild von Marcus Aurelius zum Ausdruck gebracht wird. Deshalb wäre es nicht überraschend, wenn auf schrittweise besiedeltem Gebiet durch die nordkarpathischen Einwohner diese hier zufällig entdeckten älteren Prägungen der römischen Münzen trafen. Diese müssen nicht immer ein langfristig gesammeltes Eigentum repräsentieren, sondern es kann sich auch um Münzen handeln, die einfach verlorengegangen sind oder absichtlich im Wirbel der Kriegskonflikte und auf wichtigen Straßen, Handelszügen versteckt wurden. Es ist zweifellos, dass die germanischen Stämme auch römische Exemplare, ausgefertigt aus Kupfer/Bronze, nicht verachtet haben, die auch wenn in einem geringen Ausmaß, hier vertreten sind. Auf die Tatsache, dass es sich bei den Münzen aus Spišská Belá berechtigt um römische Münzen handelt, machen nicht nur markante spätrömische Artefakte aus einzelnen Fundstätten, begleitet von abgewetzten spätrömischen Münzen aufmerksam, sondern auch eine auffällig dichte Kumulation der nordkarpathischen Besiedlung im Extravillan der heutigen Stadt, wo wir ungefähr 15 Lokalitäten der Nordkarpathischen Gruppe vermerken (GiertlováKučerová/Soják 2005, 118 f.; Soják 2006a, 41). Auf einer der behandelnden Lokalitäten mit Vorkommen von zwei hier präsentierten römischen Münzen (Hoher Rand) wurde im Jahr 2007 eine archäologische Ausgrabung, orientiert auf die mesolithische Besiedlung der Lokalität, realisiert. In der Sonde belegte man ein vertieftes Objekt, in dem neben urzeitlichen Artefakten auch ein Tierknochen und

\footnotetext{
3 Für Konsultationen zu den Fibelfunden danke ich K. Pieta.
} 
Holzkohle aufgetreten sind. Die ${ }^{14} \mathrm{C}$-Analyse der beiden organischen Reste wies auf die ältere Römerzeit (1870 \pm 30 BP, $1915 \pm 35$ BP) hin, wobei während der Rekognoszierung auf der Fundstelle auch Scherben der Nordkarpathischen Gruppe gefunden wurden (Valde-Nowak/Soják 2010, 4, Abb. 5; 6 ).

Eine analogische Situation mit dem Auftreten von älteren römischen Münzen in spätrömischer Umgebung registrieren wir aus anderen Regionen der Slowakei (Selce, Turík u. a.). Ein bedeutender Fund der den Gebrauch und vielleicht auch den Umlauf von alten römischen Denaren in der jüngeren Römerzeit (aus dem Ende des 3. bis Verlauf des 4. Jh. n. Chr.) belegt, stammt aus dem Areal der germanischen Siedlung in Bratislava-Trnávka, wo man in der Verfüllung einer quadischen Grubenhütte 13 verstreute Denare von Nero bis Marcus Aurelius fand (Varsik 2002, 43-45). Ähnliche Fundkomplexe stammen auch aus Kleinpolen (z. B. Befunde aus den Fundstellen Zagórzyn oder Świlcza; TIR 2002, 356 ff., Nr. 884; bzw. 319, Nr. 775; Kunisz 1969; 1985), weiter aus der Ukraine oder dem oberschlesischen Gebiet (Kolníková/Pieta 2009). Gleichermaßen gibt es auch auf dem Gebiet von Tschechien eine Reihe von Belegen, dass die Denare aus den 1.-2. Jh. n. Chr. noch im 5. Jh. n. Chr., also in der Völkerwanderungszeit massenhaft im Umlauf waren (Militký 2013, 38 ff.).

Die repräsentierten numismatischen Funde sind Zeugen der Tatsache, dass man die Bedeutung der römischen Münzenfunde nicht nur auf ihren Datierungswert einschränken kann, ohne Rücksicht auf den breiteren wirtschaftlich-gesellschaftlichen Grad der heimischen barbarischen Fundumgebung (Kolníková 1986). Sie sind Zeugen einer langzeitigen Anhäufung von hochwertigen römischen Prägungen, die genügend geeignetes Silbermetall enthielten, um den Trägern der Nordkarpathischen Gruppe zur Produktion von verschiedenen Metallprodukten dienen zu können, unter welchen die größte Bedeutung bestimmt Fibeln, Schnallen seltener auch Werkzeuge hatten. ${ }^{4}$

\section{LITERATUR}

Bursche 1994 - A. Bursche: Die Markomannenkriege und der Zufluß römischer Münzen in das Barbaricum. In: H. Friesinger/J. Tejral/A. Stuppner (Hrsg.): Markomannenkriege-Ursachen und Wirkungen. Spisy Archeologického ústavu AV ČR Brno 1. Brno 1994, 471-485.

Daňová/Soják 2013 - M. Daňová/M. Soják: Unikátny nález antickej gemy zo Spišskej Novej Vsi - polohy Smižianska roveň. Zborník SNM 107. Archeológia 23, 2013, 61-66.

Giertlová-Kučerová/Soják 2005 - M. Giertlová-Kučerová/ M. Soják: Novšie nálezy severokarpatskej skupiny v povodí rieky Poprad. Študijné zvesti AU SAV 38, 2005, 113-136.

Jakab 2005 - J. Jakab: Kostra muža s poúrazovými zmenami z doby rímskej z jaskyne pri Žehre. Východoslovenský pravek 7, 2005, 101-106.

Kollárová/Novák/Soják 2015 -Z. Kollárová/A. Novák/M. Soják: Dr. Michal Greisiger. Kežmarok 2015.

Kolníková 1972 - E. Kolníková: K interpretácii nálezov rímskych mincí na Slovensku. Slovenská numizmatika 2, 1972, 7-114.

Kolníková 1973 - E. Kolníková: Ku konfrontácii nálezov mincí s výsledkami bádania o dobe rímskej na Slovensku. Slovenská archeológia 21, 1973, 167-186.

Kolníková 1980 - E. Kolníková: Rímske mince na Slovensku. Ars Slovaca Antiqua. Dávnoveké umenie Slovenska 10. Bratislava 1980.

Kolníková 1986 - E. Kolníková: Kritický rozbor a klasifikácia nálezov rímskych mincí na Slovensku. Slovenská numizmatika 9, 1986, 59-97.

Kolníková 1994 - E. Kolníková: Die Markomannenkriege im Lichte der Fundmünzen aus der Slowakei. In: H. Friesinger/J. Tejral/A. Stuppner (Hrsg.): MarkomannenkriegeUrsachen und Wirkungen. Spisy Archeologického ústavu AV ČR Brno 1. Brno 1994, 487-496.
Kolníková/Pieta 2009 - E. Kolníková/K. Pieta: Spätrömische und völkerwanderungszeitliche Münzhorte und andere Münzfunde im Nordkarpatenraum In: M. Woloszyn (ed.): Byzantine Coins in Central Europe between the $5^{\text {th }}$ and $10^{\text {th }}$ century. Kraków 2009, 117-132.

Kunisz 1969 - A. Kunisz: Chronologia naptywu pieniadza rzymskiego na ziemie Małopolski. Wrocław - Warszawa Kraków 1969.

Kunisz 1985 - A. Kunisz: Znaleziska monet rzymskich z Małopolski. Wrocław - Warszawa-Kraków-Gdańsk Łódź 1985.

Lau/Pieta 2014 - N. Lau/K. Pieta: Das Grab von PopradMatejovce in der Slowakei - Anlage, Konstruktion und Ausstattung eines frühvölkerwanderungszeitlichen Kammergrabes. In: A. Abegg-Wigg/N. Lau (Hrsg.): Kammergräber im Barbaricum. Zu Einflüssen und Übergangsphänomenen von der vorrömischen Eisenzeit bis in die Völkerwanderungszeit. Internationale Tagung. Schleswig, 25.-27. November 2010. Schriften des Archäologischen Landesmuseums Ergänzungsreihe 9. Neumünster Hamburg 2014, 343-364.

Lipták 1935 - J. Lipták: Urgeschichte und Besiedlung der Zips. Bilder aus der Zipser Vergangenheit. Kesmark 1935.

Militký 2013 - J. Militký: Depoty a hromadné nálezy římských a raně byzantských mincí. In: L. Smejtek/M. Lutovský/J. Militký: Encyklopedie pravěkých pokladů v Čechách. Praha 2013, 36-42.

Minarovičová 1996 - E. Minarovičová: Keltské a rímske mince zo súkromnej zbierky Michala Greisigera. Zborník SNM 90. História 36, 1996, 131-141.

Novotná/Soják 2013 - M. Novotná/M. Soják: Vel’ká LomnicaBurchbrich. Urzeitliches Dorf unter den Hohen Tatra. Archaeologica Slovaca Monographiae. Studia 16. Nitra 2013.

\footnotetext{
4 Zu erwähnen ist auch z. B. ein silberner Pfriem aus einem Kammergrab in Poprad-Matejovce (Lau/Pieta 2014, 360, Abb. 17).
} 
Pieta 1991 - K. Pieta: The North Carpathians at the Beginning of the Migration Period. Antiquity 65 (247), 1991, 376-387.

RIC II 1968 - H. Mattingly/E. A. Sydenham: The Roman Imperial Coinage. Volume II. Vespasian to Hadrian. London 1968.

RIC III 1968 - H. Mattingly/E. A. Sydenham: The Roman Imperial Coinage. Volume III. Antoninus Pius to Commodus. London 1968.

Šmálik 1967 - Š. Šmálik: Nález rímskej mince pri Spišskom Podhradí. Nové obzory 9, 1967, 208-210.

Soják 1998 - M. Soják: Rímska minca z Vlkovej-Levkoviec. Slovenská numizmatika 15, 1998, 225-227.

Soják 2000 - M. Soják: Nálezy rímskych mincí na Spiši: problém riešenia rímsko-barbarských vztahov na základe numizmatických prameňov. Numizmatika 17 supplementum, 2000, 97-104.

Soják 2002 - M. Soják: Nález rímskej mince zo Strání pod Tatrami. Slovenská numizmatika 16, 2002, 184-186.

Soják 2003a-M. Soják: Prieskum a výskum v oblasti Spiša. AVANS 2002, 2003, 132-142.

Soják 2003b - M. Soják: Rašelinisko Trstinné lúky pri Spišskej Belej v kontexte pravekého a včasnodejinného osídlenia. Východoslovenský pravek 6, 2003, 167-173.

Soják 2004 - M. Soják: Traianov denár z Hôrky-Primoviec. Slovenská numizmatika 17, 2004, 195, 196.

Manuskript angenommen am 22. 9. 2021

Übersetzt von Dominika Boháčová

Abstract translated by Lucia Benediková

PhDr. Marián Soják, PhD.

Archeologický ústav SAV

Oddelenie záchranných výskumov - Spiš

Mlynská 6

SK - 05201 Spišská Nová Ves

sojak@ta3.sk
Soják 2005a-M. Soják: Die Entdeckung römischer Münzen und menschlicher Knochen in der Höhle Temná, Gemeinde Žehra, Nordslowakei. Archäologisches Korrespondenzblatt 35, 2005, 77-87.

Soják 2005b - M. Soják: Osídlenie Temnej jaskyne pod Spišským hradom. Východoslovenský pravek7, 2005, 83-100.

Soják 2006a-M. Soják: Pred prvou písomnou zmienkou. In: Z. Kollárová (zost.): Spišská Belá. Prešov 2006, 23-51, 130.

Soják $2006 b$ - M. Soják: Unikátny nález z doby rímskej pod Spišským hradom. Acta Musaei Scepusiensis 2006. Pohlady do minulosti 6. Levoča 2006, 9-24.

Soják 2008 - M. Soják: Výpoved' nálezov antických mincí zo Spiša. Zborník SNM 100. História 46, 2008, 31-44.

Soják 2014 - M. Soják: Spišská Nová Ves v archeologických svedectvách. In: I. Chalupecký (zost.): Dejiny mesta Spišská Nová Ves. Spišská Nová Ves 2014, 32-65.

Soják/Fecko 2015 - M. Soják/P. Fecko: Ojedinelý nález rímskej mince z Janoviec pri Bardejove. Slovenská numizmatika 20, 2015, 171-173.

TIR 2002 - P. Kaczanowski/U. Margos (eds.): Tabula Imperii Romani. M 34-Kraków. Kraków 2002.

Valde-Nowak/Soják 2010 - P. Valde-Nowak/M. Soják: Contribution to the Mesolithic in the Slovak Carpathians. Slovenská archeológia 58, 2010, 1-12.

Varsik 2002 - V. Varsik: Rímske mince z Bratislavy-Trnávky. Slovenská numizmatika 16, 2002, 41-50.

MUDr. Andrej Novák

Mierová 9

SK - 05901 Spišská Belá

novak.mudr@gmail.com 